\title{
Psychological Factors, Coffee and Risk of Diabetes Mellitus among Middle-Aged Japanese: a Population-Based Prospective Study in the JPHC Study Cohort
}

\author{
Masayuki KATO*, Mitsuhiko NODA**, Manami INOUE***, TAKAShi KADOWAKI ${ }^{*}$, \\ SHoichiro TSUGANE***; for the JPHC Study Group \\ * Japan Foundation for the Promotion of International Medical Research Cooperation, 1-21-13 Toyama, Shinjuku-ku, Tokyo 162- \\ 0052, Japan \\ ** Department of Diabetes and Metabolic Medicine, International Medical Center of Japan, 1-21-1 Toyama, Shinjuku-ku, Tokyo \\ 162-8655, Japan \\ ***Epidemiology and Prevention Division, Research Center for Cancer Prevention and Screening, National Cancer Center, 5-1-1 \\ Tsukiji, Chuo-ku, Tokyo 104-0045, Japan \\ \# Department of Metabolic Diseases, Graduate School of Medicine, University of Tokyo, 7-3-1 Hongo, Bunkyo-ku, Tokyo 113-8655, Japan
}

\begin{abstract}
An association between psychological factors and diabetes has been suspected for a long time. However, epidemiological data on this association is limited. We investigated the association between psychological factors (perceived mental stress and type A behavior) and the onset of diabetes in a community-based, prospective cohort study in a large number of middle-aged Japanese adults. A total of 55,826 subjects (24,826 men and 31,000 women) aged 40-69 years were followed for 10 years. A self-administered questionnaire on medical conditions including diabetes and other lifestyle factors was performed at baseline and 5 and 10 years later. Psychological factors and diabetes were assessed based on the questionnaire results. During the 10-year follow-up period, we documented 1,601 incident cases (6.4\%) of diabetes among men and 1,093 cases (3.5\%) among women. The risk of diabetes increased with an increasing stress level, especially among men. Multivariate adjusted odds ratios for high stress compared with low stress were 1.36 (1.13 to 1.63) among men and 1.22 ( 0.98 to 1.51 ) among women. The risk of diabetes increased with an increasing level of type A behavior only among women. Multivariate adjusted odds ratios for high levels of type A behavior compared with low levels of type A behavior were 1.09 (0.94 to 1.27) among men and 1.22 (1.01 to 1.47) among women. We found an association between perceived mental stress and the incidence of diabetes, especially among men. We also found an association between type A behavior and the incidence of diabetes among women. In addition, inverse association between coffee consumption and the incidence of diabetes which was consistent with other studies was observed.
\end{abstract}

Key words: diabetes mellitus, perceived mental stress, type A behavior, coffee

(Endocrine Journal 56: 459-468, 2009)

THE PREVALENCE of type 2 diabetes has increased dramatically over the past few decades. Recent estimates indicate that there were 171 million people with diabetes worldwide in the year 2000 and this number

Received: January 5, 2009

Accepted: February 3, 2009

Correspondence to: Mitsuhiko NODA, Department of Diabetes and Metabolic Medicine, International Medical Center of Japan, 1-21-1 Toyama, Shinjuku-ku, Tokyo 162-8655, Japan Abbreviations: HPA, hypothalamus-pituitary-adrenal; JPHC study, The Japan Public Health Centre-based prospective Study; IL-6, interleukin-6; OR, odds ratio; 95\% CI, 95\% confidence interval. is projected to increase to 366 million by 2030 [1]. Diabetes is now one of the main threats to human health and is likely to remain a huge threat to public health in years to come [2].

An association between psychological factors and diabetes has long been suspected $[3,4]$. For example, mental stress is thought to deteriorate glucose metabolism through the activation of the hypothalamuspituitary-adrenal axis (HPA axis) and the sympathetic nervous system [5-8]. Type A behavior pattern is also thought to activate the HPA axis and the sympathetic nervous system. However, epidemiological data about 
the association between these psychological factors and diabetes is limited [9-13], and, as far as we know, no study has shown an association between these factors and the incidence of diabetes in a general population. In this paper, we examined the effect of psychological factors (mental stress and type A behavior) on the incidence of diabetes mellitus in a communitybased, prospective cohort study in a large number of middle-aged Japanese adults.

\section{Materials and Methods}

\section{Subjects and procedures}

The Japan Public Health Centre-based prospective Study (JPHC Study) is an ongoing, longitudinal cohort study investigating cancer, cardiovascular diseases and other lifestyle-related diseases. The JPHC Study was launched in 1990 for cohort I and in 1993 for cohort II. Cohort I was composed of five prefectural public health center areas; Ninohe (Iwate Prefecture), Yokote (Akita Prefecture), Saku (Nagano Prefecture), Chubu (Okinawa Prefecture), and Katsushika (metropolitan Tokyo). Cohort II was composed of six prefectural public health center areas; Mito (Ibaraki Prefecture), Nagaoka (Nigata Prefecture), Chuohigashi (Kochi Prefecture), Kamigoto (Nagasaki Prefecture), Miyako (Okinawa Prefecture), and Suita (Osaka Prefecture). The details of the study design have been described elsewhere [14]. The study protocol was approved by the institutional review board of the National Cancer Center, Japan. In the present analysis, two public health center areas (Katsushika and Suita) were excluded because different definitions for the study population were applied. The study population was defined as all registered Japanese inhabitants of the nine public health center areas aged 40-59 years (Cohort I) and 40-69 years (Cohort II) at the beginning of each baseline survey. Each participant completed a self-administered questionnaire that included questions about weight, height, previously diagnosed medical conditions, family history of diabetes, use of drugs, and other lifestyle factors such as physical activity and smoking. This questionnaire also included a food frequency questionnaire that was validated using 28-day diet records [15]. The questionnaire was performed at baseline and 5 and 10 years later. Of the 95,373 individuals (45,452 men and 49,921 women) who completed the baseline questionnaire upon enrolment, 71,713 (75\%, 32,369 men and 39,344 women) responded to both of the follow-up questionnaires. We excluded individuals who had any of the following conditions at baseline; cardiovascular disease, chronic liver disease, kidney disease or any type of cancer $(n=4,515)$. Individuals who had missing baseline data for any of the exposure parameters described below were also excluded $(n=9,256)$. Individuals with a body mass index (calculated as weight in kilograms divided by the square of height in meters) of less than 14 or more than 40 were also excluded because of the possibility of unreliable data $(n=741)$. Because the present study examined the incidence of diabetes, we also excluded any subjects with diabetes at baseline $(n=3,092)$. After these exclusions, the remaining cohort consisted of 55,826 participants $(24,826$ men and 31,000 women $)$.

\section{Assessment of Psychological factors and diabetes}

Mental stress was assessed based on three levels of response (low, medium and high) to the question, 'How much stress do you feel in your daily life?' We assessed four aspects of the type A behavioral pattern through self-reports of: competitive drive, speed and impatience, aggressiveness and irritability [16]. These items were assessed by the level (very, somewhat, or not at all) for each question: 'How hasty and impatient do you consider yourself to be?', 'How competitive and eager to excel in everything do you consider yourself to be?', 'How aggressive do you consider yourself to be?', and 'How irritable do you consider yourself to be?'. The above four items were scored from 0 ("not at all") to 2 ("very") and then combined into an overall index of type A behavior pattern. These items matched well with validated instruments such as the Framingham Type A Scale and MMPI-2 Type A Scale in domains such as aggression, irritability, competitiveness and time urgency [16].

We defined subjects with diabetes (diagnosed diabetes) as those who answered 'yes' to the question 'Has a doctor ever told you that you have diabetes?' or 'Do you take any anti-diabetic drugs?'. To document the validity of the diagnosed diabetes, we examined a series of medical records: $94 \%$ of the cases of diagnosed diabetes according to the questionnaire were confirmed by medical records [17]. We also conducted a cross-sectional survey in 1990 to examine the sensitivity of diagnosed diabetes according to the criteria 
Table 1a. Baseline characteristics of the analysis group according to stress levels.

\begin{tabular}{|c|c|c|c|c|c|c|}
\hline & \multirow{2}{*}{\multicolumn{3}{|c|}{$\begin{array}{c}\text { Men }(\mathrm{n}=24,826) \\
\text { Perceived Mental Stress }\end{array}$}} & \multirow{2}{*}{\multicolumn{3}{|c|}{$\begin{array}{c}\text { Women }(\mathrm{n}=31,000) \\
\text { Perceived Mental Stress }\end{array}$}} \\
\hline & & & & & & \\
\hline & $\begin{array}{c}\text { Low } \\
(n=3,405)\end{array}$ & $\begin{array}{c}\text { Medium } \\
(\mathrm{n}=15,734)\end{array}$ & $\begin{array}{c}\text { High } \\
(\mathrm{n}=5,687)\end{array}$ & $\begin{array}{c}\text { Low } \\
(\mathrm{n}=4,865)\end{array}$ & $\begin{array}{c}\text { Medium } \\
(\mathrm{n}=20,416)\end{array}$ & $\begin{array}{c}\text { High } \\
(\mathrm{n}=5,719)\end{array}$ \\
\hline Age & 53.3 & 51.4 & 48.2 & 52.9 & 51.6 & 49.1 \\
\hline Body Mass Index & 23.7 & 23.5 & 23.5 & 23.6 & 23.5 & 23.2 \\
\hline Current smoker & 49.2 & 50.2 & 55.0 & 3.9 & 3.9 & 6.8 \\
\hline Alcohol drinkers* & 66.1 & 67.4 & 70.1 & 10.1 & 9.5 & 14.0 \\
\hline Alcohol intake among drinkers (g/week) & 270.9 & 264.9 & 265.9 & 79.6 & 79.0 & 88.4 \\
\hline Family history of diabetes (yes) & 8.3 & 7.9 & 10.6 & 8.6 & 8.4 & 10.4 \\
\hline Physical activity (yes) & 22.0 & 18.3 & 17.1 & 22.0 & 16.6 & 13.0 \\
\hline History of hypertension (yes) & 17.3 & 17.2 & 16.7 & 16.0 & 16.5 & 14.9 \\
\hline Coffee ( $\geq 3$ cups/day) & 12.3 & 11.5 & 19.1 & 8.6 & 7.9 & 13.3 \\
\hline Hours of sleep & 7.6 & 7.5 & 7.2 & 7.3 & 7.2 & 6.9 \\
\hline \multicolumn{7}{|l|}{ Level of Type A behavior pattern index } \\
\hline 1 (most Type A) & 21.3 & 22.1 & 36.1 & 12.1 & 13.6 & 26.5 \\
\hline 2 & 15.7 & 16.2 & 16.8 & 13.6 & 15.3 & 18.7 \\
\hline 3 & 35.2 & 42.0 & 28.2 & 39.3 & 46.7 & 30.9 \\
\hline 4 (most Type B) & 27.8 & 19.7 & 18.9 & 35.0 & 24.5 & 23.9 \\
\hline
\end{tabular}

Age, body mass index, alcohol intake and hours of sleep are represented as the mean, and the other variables are proportion (\%).

*Alcohol drinkers: drinking alcohol $\geq 1$ day/week

at that time for subjects (health checkup participants) whose plasma glucose data were available. Among the 6,118 subjects, 248 subjects had diagnosed diabetes. Among the 5,927 subjects who did not have diagnosed diabetes, 49 subjects $(0.83 \%)$ had diabetes according to the commonly used diagnostic standards utilized in Japan in 1990 (fasting plasma glucose $\geq$ $7.8 \mathrm{mmol} / \mathrm{L}$; casual plasma glucose $\geq 11 \mathrm{mmol} / \mathrm{L}$ ) [18] based on a single measurement. Taking into account the above mentioned positive predictive value, the sensitivity and specificity of diagnosed diabetes were $82.9 \%$ and $99.7 \%$, respectively.

Those who did not have diagnosed diabetes at baseline but who had it at the time of either the 5- or 10year follow-up questionnaire were defined as incident cases of diabetes.

\section{Statistical Analysis}

All analyses were performed using the data from the 55,826 individuals $(24,826$ men and 31,000 women) who responded to both the 5- and 10-year questionnaires. The cumulative incidence was defined as the number of new cases of diabetes occurring during the 10-year follow up period divided by the number of subjects at risk of developing diabetes at baseline. We carried out a multivariate analysis using logistic regression to assess the risk of diabetes as odds ratios adjusted for potential confounding factors such as age (continuous), body mass index (continuous), smoking status (never smoker, past smoker, or current smoker at $<20$ or $\geq 20$ cigarettes per day), alcohol intake (nonor infrequent occasional drinkers, or regular drinkers categorized according to weekly alcohol intake), family history of diabetes (at least one parent or one sibling with diabetes), physical activity (participation in sports at least once a week), history of hypertension, and coffee consumption $(<3$ or $\geq 3$ cups of coffee per day). Type A behavioral pattern was categorized into four groups based on the overall index of type A behavior pattern: very high (scores of 6-8), high (score of 5), medium (score of 4) or low (scores of 0-3). Hours of sleep was also included in the multivariate analysis as categorical variables $(\leq 5,6,7,8$, and $\geq$ 9 hours) because this factors may be associated with psychological factors. All analyses were performed separately for men and women.

\section{Results}

The baseline characteristics of the analyzed subjects according to stress levels and type A behavioral pattern index are shown in Table $1 \mathrm{a}$ and $1 \mathrm{~b}$, respec- 
Table 1b. Baseline characteristics of the analysis group according to levels of Type A behavior pattern index.

\begin{tabular}{|c|c|c|c|c|c|c|c|c|}
\hline & \multicolumn{4}{|c|}{ Men $(n=24,826)$} & \multicolumn{4}{|c|}{ Women $(n=31,000)$} \\
\hline & \multicolumn{4}{|c|}{ Levels of Type A behavior pattern index } & \multicolumn{4}{|c|}{ Levels of Type A behavior pattern index } \\
\hline & $\begin{array}{c}1 \text { (most Type A) } \\
(\mathrm{n}=6,253)\end{array}$ & $\begin{array}{c}2 \\
(n=4,043)\end{array}$ & $\begin{array}{c}3 \\
(n=9,408)\end{array}$ & $\begin{array}{c}4 \\
(n=5,122)\end{array}$ & $\begin{array}{c}1 \\
(n=4,886)\end{array}$ & $\begin{array}{c}2 \\
(\mathrm{n}=4,847)\end{array}$ & $\begin{array}{c}3 \\
(\mathrm{n}=13,206)\end{array}$ & $\begin{array}{c}4 \\
(n=8,061)\end{array}$ \\
\hline Age & 50.4 & 50.8 & 51.5 & 50.8 & 50.5 & 50.9 & 52.0 & 51.1 \\
\hline Body Mass Index & 23.6 & 23.5 & 23.5 & 23.5 & 23.3 & 23.3 & 23.4 & 23.7 \\
\hline Current smoker & 53.2 & 51.5 & 51.1 & 48.7 & 7.3 & 4.7 & 3.7 & 3.7 \\
\hline Alcohol drinkers* & 71.4 & 69.2 & 67.4 & 64.2 & 14.5 & 12.1 & 8.8 & 9.5 \\
\hline $\begin{array}{l}\text { Alcohol intake among } \\
\text { drinkers (g/week) }\end{array}$ & 290.5 & 276.0 & 250.9 & 253.3 & 104.7 & 76.5 & 72.3 & 77.6 \\
\hline $\begin{array}{l}\text { Family history of } \\
\text { diabetes (yes) }\end{array}$ & 9.0 & 8.8 & 7.8 & 9.2 & 10.1 & 9.1 & 8.2 & 8.8 \\
\hline Physical activity (yes) & 19.8 & 20.3 & 18.0 & 16.5 & 16.9 & 18.0 & 16.8 & 15.9 \\
\hline $\begin{array}{l}\text { History of } \\
\text { hypertension (yes) }\end{array}$ & 19.2 & 16.9 & 16.5 & 15.8 & 15.7 & 16.6 & 16.4 & 15.8 \\
\hline Coffee ( $\geq 3$ cups/day) & 15.0 & 13.1 & 12.3 & 13.4 & 11.3 & 10.1 & 8.2 & 8.4 \\
\hline Hours of sleep & 7.4 & 7.5 & 7.4 & 7.4 & 7.1 & 7.2 & 7.2 & 7.1 \\
\hline \multicolumn{9}{|l|}{ Perceived Mental Stress } \\
\hline Low & 11.6 & 13.2 & 12.7 & 18.5 & 12.1 & 13.7 & 14.5 & 21.1 \\
\hline Medium & 55.6 & 63.1 & 70.2 & 60.6 & 56.9 & 64.3 & 72.1 & 61.9 \\
\hline High & 32.8 & 23.7 & 17.1 & 20.9 & 31.0 & 22.0 & 13.4 & 17.0 \\
\hline
\end{tabular}

Age, body mass index, alcohol intake and hours of sleep are represented as the mean, and the other variables are proportion (\%).

*Alcohol drinkers: drinking alcohol $\geq 1$ day/week

tively. Compared with the subjects who reported low stress, those who reported higher stress were likely to be younger, smokers, physically inactive and coffee drinkers. Compared with the subjects with low levels of type A index, those with higher levels of type A index were likely to be smokers, alcohol drinkers and coffee drinkers.

During the 10-year follow-up period, we documented 1,601 incident cases (6.4\%) of diabetes among men and 1,093 cases $(3.5 \%)$ among women. The age- and multivariate-adjusted odds ratios for the incidences of diabetes mellitus according to perceived mental stress are shown in Table 2. For men, a significant, dose-response association was observed between perceived mental stress and diabetes. This association remained almost unchanged after adjustments for known risk factors of diabetes, type A and hours of sleep. For women, the risk of diabetes also increased as the stress level increased. However, this association and doseresponse relation were slightly weakened after adjustments for known risk factors of diabetes and margin- ally disappeared after further adjustments for type A and hours of sleep.

The age- and multivariate-adjusted odds ratios for the incidences of diabetes mellitus according to type A index are shown in Table 3. For men, association between type A index and the risk of diabetes was not observed after adjustments for other risk factors of diabetes. For women, risk of diabetes was higher for those with highest level of type A behavior and this association was slightly strengthened after adjustments for known risk factors of diabetes, perceived mental stress and hours of sleep.

As for individual components of type A behavior pattern, high level of impatience, irritability and aggressiveness were associated, although not statistically significant, with the risk of diabetes (Table 4).

Recently, a number of papers have reported the protective effect of coffee against diabetes, and we included coffee consumption as a known risk factor of diabetes in the above analysis. In fact, coffee consumption ( $\geq 3$ cups of coffee/day) reduced the risk of diabe- 
Table 2. Odds ratios for the 10 -year incidences of diabetes mellitus according to perceived mental stress Men

\begin{tabular}{|c|c|c|c|c|c|c|c|}
\hline & \multicolumn{6}{|c|}{ Perceived Mental Stress } & \\
\hline & & $\begin{array}{c}\text { Low } \\
(n=3,405)\end{array}$ & & $\begin{array}{l}\text { Medium } \\
(\mathrm{n}=15,734)\end{array}$ & & $\begin{array}{c}\text { High } \\
(\mathrm{n}=5,687)\end{array}$ & \\
\hline Cases & & 199 & & 999 & & 403 & \\
\hline Odds ratio $(95 \% \mathrm{CI})$ & & & & & & & $\mathrm{p}$ for trend \\
\hline Age-adjusted OR & 1 & (reference) & 1.14 & $(0.97-1.33)$ & 1.38 & $(1.15-1.65)$ & $<0.001$ \\
\hline Multivariate $\mathrm{OR}^{1}$ & 1 & (reference) & 1.20 & $(1.02-1.41)$ & 1.39 & $(1.16-1.67)$ & $<0.001$ \\
\hline Multivariate $\mathrm{OR}^{2}$ & 1 & (reference) & 1.19 & $(1.01-1.40)$ & 1.36 & $(1.13-1.63)$ & 0.001 \\
\hline
\end{tabular}

Women

\begin{tabular}{|c|c|c|c|c|c|c|c|}
\hline & & & Per & ceived Mental & & & \\
\hline & & $\begin{array}{c}\text { Low } \\
(n=4,865)\end{array}$ & & $\underset{(n=20,416)}{\text { Medium }}$ & & $\begin{array}{c}\text { High } \\
(\mathrm{n}=5,719)\end{array}$ & \\
\hline Cases & & 163 & & 720 & & 210 & \\
\hline Odds ratio $(95 \% \mathrm{CI})$ & & & & & & & $\mathrm{p}$ for trend \\
\hline Age-adjusted OR & 1 & (reference) & 1.11 & $(0.94-1.32)$ & 1.28 & $(1.04-1.58)$ & 0.020 \\
\hline Multivariate $\mathrm{OR}^{1}$ & 1 & (reference) & 1.11 & $(0.93-1.33)$ & 1.25 & $(1.01-1.56)$ & 0.038 \\
\hline Multivariate $\mathrm{OR}^{2}$ & 1 & (reference) & 1.12 & $(0.94-1.34)$ & 1.22 & $(0.98-1.51)$ & 0.080 \\
\hline
\end{tabular}

Multivariate $\mathrm{OR}^{1}$ : adjusted for age + other known risk factors of diabetes (body mass index, smoking status, alcohol drinking, family history of diabetes, physical activity, history of hypertension and coffee consumption)

Multivariate $\mathrm{OR}^{2}$ : adjusted for age + other known risk factors of diabetes + levels of Type A behavior and hours of sleep.

Table 3. Odds ratios for the 10-year incidences of diabetes mellitus according to levels of Type A behavior pattern index Men

\begin{tabular}{|c|c|c|c|c|c|c|c|c|c|}
\hline & \multicolumn{8}{|c|}{ Levels of Type A behavior pattern $(4=$ most Type B, $1=$ most Type A) } & \\
\hline & & $\begin{array}{c}4 \\
(n=5,122)\end{array}$ & & $\begin{array}{c}3 \\
(n=9,408)\end{array}$ & & $\begin{array}{c}2 \\
(\mathrm{n}=4,043)\end{array}$ & & $\begin{array}{c}1 \\
(\mathrm{n}=6,253)\end{array}$ & \\
\hline Cases & & 313 & & 599 & & 249 & & 440 & \\
\hline Odds ratio $(95 \% \mathrm{CI})$ & & & & & & & & & $\mathrm{p}$ for trend \\
\hline Age-adjusted OR & 1 & (reference) & 1.03 & $(0.90-1.19)$ & 1.01 & $(0.85-1.20)$ & 1.17 & $(1.01-1.36)$ & 0.038 \\
\hline Multivariate $\mathrm{OR}^{1}$ & 1 & (reference) & 1.06 & $(0.92-1.23)$ & 1.02 & $(0.85-1.21)$ & 1.12 & $(0.96-1.31)$ & 0.20 \\
\hline Multivariate $\mathrm{OR}^{2}$ & 1 & (reference) & 1.06 & $(0.91-1.22)$ & 1.00 & $(0.84-1.20)$ & 1.09 & $(0.94-1.27)$ & 0.381 \\
\hline
\end{tabular}

Women

\begin{tabular}{|c|c|c|c|c|c|c|c|c|c|}
\hline & \multicolumn{8}{|c|}{ Levels of Type A behavior pattern $(4=$ most Type B, $1=$ most Type A) } & \\
\hline & & $\begin{array}{c}4 \\
(n=8,061)\end{array}$ & & $\begin{array}{c}3 \\
n=13,206)\end{array}$ & & $\begin{array}{c}2 \\
(\mathrm{n}=4,847)\end{array}$ & & $\begin{array}{c}1 \\
(\mathrm{n}=4,886)\end{array}$ & \\
\hline Cases & & 292 & & 428 & & 169 & & 204 & \\
\hline Odds ratio $(95 \% \mathrm{CI})$ & & & & & & & & & $\mathrm{p}$ for trend \\
\hline Age-adjusted OR & 1 & (reference) & 0.86 & $(0.74-1.00)$ & 0.97 & $(0.80-1.18)$ & 1.19 & $(0.99-1.43)$ & 0.044 \\
\hline Multivariate $\mathrm{OR}^{1}$ & 1 & (reference) & 0.93 & $(0.80-1.09)$ & 1.05 & $(0.86-1.27)$ & 1.25 & $(1.03-1.50)$ & 0.014 \\
\hline Multivariate $\mathrm{OR}^{2}$ & 1 & (reference) & 0.93 & $(0.79-1.09)$ & 1.03 & $(0.85-1.26)$ & 1.22 & $(1.01-1.47)$ & 0.031 \\
\hline
\end{tabular}

Multivariate $\mathrm{OR}^{1}$ : adjusted for age + other known risk factors of diabetes (body mass index, smoking status, alcohol drinking, family history of diabetes, physical activity, history of hypertension and coffee consumption)

Multivariate $\mathrm{OR}^{2}$ : adjusted for age + other known risk factors of diabetes + perceived mental stress and hours of sleep. 
Table 4. Odds ratios for the 10-year incidences of diabetes mellitus according to levels of constituent items of Type A behavior pattern index

Men

\begin{tabular}{|c|c|c|c|c|c|c|c|}
\hline & & & Low & & Medium & & High \\
\hline \multirow[t]{3}{*}{ Impatience } & $\mathrm{n}$ & & 2,932 & & 14,829 & & 7,065 \\
\hline & cases & & 195 & & 942 & & 464 \\
\hline & Odds ratio $(95 \% \mathrm{CI})$ & 1 & (reference) & 1.01 & $(0.85-1.18)$ & 1.02 & $(0.86-1.22)$ \\
\hline \multirow[t]{3}{*}{ Irritability } & $\mathrm{n}$ & & 3,024 & & 14,584 & & 7,218 \\
\hline & cases & & 179 & & 921 & & 501 \\
\hline & Odds ratio $(95 \% \mathrm{CI})$ & 1 & (reference) & 1.08 & $(0.92-1.28)$ & 1.14 & $(0.95-1.37)$ \\
\hline \multirow[t]{3}{*}{ Aggressiveness } & $\mathrm{n}$ & & 2,885 & & 17,118 & & 4,823 \\
\hline & cases & & 167 & & 1,079 & & 355 \\
\hline & Odds ratio $(95 \% \mathrm{CI})$ & 1 & (reference) & 1.05 & $(0.89-1.25)$ & 1.12 & $(0.92-1.36)$ \\
\hline \multirow[t]{3}{*}{ Competitiveness } & $\mathrm{n}$ & & 2,405 & & 17,388 & & 5,033 \\
\hline & cases & & 172 & & 1,081 & & 348 \\
\hline & Odds ratio $(95 \% \mathrm{CI})$ & 1 & (reference) & 0.87 & $(0.73-1.03)$ & 0.90 & $(0.74-1.09)$ \\
\hline
\end{tabular}

Women

\begin{tabular}{|c|c|c|c|c|c|c|c|}
\hline & & & Low & & Medium & & High \\
\hline \multirow[t]{3}{*}{ Impatience } & $\mathrm{n}$ & & 4,391 & & 19,491 & & 7,118 \\
\hline & cases & & 160 & & 665 & & 268 \\
\hline & Odds ratio $(95 \% \mathrm{CI})$ & 1 & (reference) & 1.05 & $(0.88-1.26)$ & 1.23 & $(1.00-1.51)$ \\
\hline \multirow[t]{3}{*}{ Irritability } & $\mathrm{n}$ & & 2,931 & & 22,312 & & 5,757 \\
\hline & cases & & 107 & & 762 & & 224 \\
\hline & Odds ratio $(95 \% \mathrm{CI})$ & 1 & (reference) & 0.99 & $(0.80-1.23)$ & 1.16 & $(0.91-1.48)$ \\
\hline \multirow[t]{3}{*}{ Aggressiveness } & $\mathrm{n}$ & & 4,738 & & 21,755 & & 4,507 \\
\hline & cases & & 155 & & 757 & & 181 \\
\hline & Odds ratio $(95 \% \mathrm{CI})$ & 1 & (reference) & 0.96 & $(0.80-1.15)$ & 1.08 & $(0.87-1.36)$ \\
\hline \multirow[t]{3}{*}{ Competitiveness } & $\mathrm{n}$ & & 4,101 & & 23,261 & & 3,638 \\
\hline & cases & & 155 & & 804 & & 134 \\
\hline & Odds ratio $(95 \% \mathrm{CI})$ & 1 & (reference) & 0.99 & $(0.83-1.19)$ & 1.01 & $(0.80-1.29)$ \\
\hline
\end{tabular}

adjusted for age, other known risk factors of diabetes (body mass index, smoking status, alcohol drinking, family history of diabetes, physical activity, history of hypertension and coffee consumption), perceived mental stress and hours of sleep

Table 5. Odds ratios for the 10-year incidences of diabetes mellitus according to coffee consumption Men

\begin{tabular}{lrrrr}
\hline & $\mathrm{n}$ & cases & Odds Ratio & $(95 \% \mathrm{CI})$ \\
\hline almost never (reference) & 7,378 & 540 & 1 & \\
1-2 days per week & 4,703 & 314 & 0.93 & $(0.80-1.08)$ \\
3-4 days per week & 3,012 & 184 & 0.84 & $(0.71-1.01)$ \\
1-2 cups/day & 6,417 & 374 & 0.84 & $(0.73-0.97)$ \\
3-4 cups/day & 2,442 & 138 & 0.83 & $(0.68-1.02)$ \\
$\geq 5$ cup/day & 874 & 51 & 0.82 & $(0.60-1.11)$ \\
\hline \multicolumn{1}{c}{ p for trend } & & & 0.006 & \\
Women & & & &
\end{tabular}

\begin{tabular}{lccrc} 
Women & $\mathrm{n}$ & cases & Odds Ratio & $(95 \%$ CI $)$ \\
\hline almost never (reference) & 9,873 & 429 & 1 & \\
1-2 days per week & 5,975 & 218 & 0.90 & $(0.76-1.06)$ \\
3-4 days per week & 3,515 & 127 & 0.95 & $(0.77-1.17)$ \\
1-2 cups/day & 8,836 & 262 & 0.81 & $(0.69-0.96)$ \\
3-4 cups/day & 2,223 & 48 & 0.62 & $(0.45-0.84)$ \\
$\geq 5$ cup/day & 578 & 9 & 0.40 & $(0.20-0.78)$ \\
\hline \multicolumn{1}{c}{ p for trend } & & & $<0.001$ &
\end{tabular}

adjusted for age, other known risk factors of diabetes (body mass index, smoking status, alcohol drinking, family history of diabetes, physical activity, history of hypertension), perceived mental stress, levels of Type A behavior and hours of sleep 
tes in the present analysis, especially among women [odds ratio and 95\% confidence interval were 0.91 (0.77-1.08) for men and 0.63 (0.47-0.83) for women] and there exists dose-response relationship (Table 5). Because coffee is also thought to have a psychological effect as described later in the Discussion section, we also conducted an analysis stratified according to coffee consumption ( $<3$ or $\geq 3$ cups of coffee/day). After adjustments for all of the above-described parameters, the odds ratio (and 95\% CI) for medium and high stress compared with low stress were 1.23 (1.03-1.46) and 1.42 (1.16-1.73) for subjects consuming $<3$ cups of coffee/day $(\mathrm{p}$ for trend $=0.001)$ and $0.93(0.58-1.47)$ and $1.01(0.61-1.67)$ for subjects consuming $\geq 3$ cups of coffee/day ( $\mathrm{p}$ for trend $=0.836$ ) for men. For women, these values were $1.14(0.95-1.37)$ and $1.20(0.96-$ 1.51) for subjects consuming $<3$ cups of coffee/day (p for trend $=0.118)$ and $0.80(0.36-1.75)$ and $1.24(0.53$ 2.87) for subjects consuming $\geq 3$ cups of coffee/day ( $p$ for trend $=0.427$ ). The association between perceived mental stress and diabetes was observed only among subjects who consumed $<3$ cups of coffee/day, especially among men. No such effect modification was observed for type A behavior pattern.

\section{Discussion}

We found that perceived mental stress was positively associated with the incidence of diabetes mellitus. The association between perceived mental stress and the incidence of diabetes mellitus was evident among men and it was statistically significant after adjustments for known risk factors of diabetes and also after further adjustments for type A behavior and hours of sleep. For women, the association between perceived mental stress and the incidence of diabetes mellitus was slightly weakened after adjustments for known risk factors of diabetes and it was marginally lower than the significant level after further adjustments for type A behavior and hours of sleep. We also found an association between type A behavior and the incidence of diabetes mellitus. In contrast to mental stress, this association was statistically significant only among women. The reason for this difference is unclear. However, one possible explanation is that there could be a difference between men and women in terms of the mutual modification between mental stress and the type A behavior, on the hypothetical basis that the overall mechanisms by which mental stress and type A behavior give rise to diabetes may be overlapping, as discussed below, and may possibly be modifying each other.

An association between mental stress and diabetes has long been suspected [3,4]. The mechanism by which mental stress causes diabetes is unclear, but the activation of two systems, the hypothalamus-pituitaryadrenal (HPA) axis and the sympathetic nervous system, are suspected to play roles $[8,19]$. Activation of the HPA axis and the sympathetic nervous system increases secretion of cortisol and catecholamines, and hence, leads to the deterioration of glucose metabolism. Interleukin-6 (IL-6), which is suspected to play a pathologic role in a range of diseases including diabetes, is secreted during stress and participates in the stress response [20]. In fact, Kiecolt-Glaser reported an association, in caregivers, between chronic stress and increased plasma levels of IL-6 [21]. Other mechanisms such as stress induced activation of inflammatory response and the failed downregulation of corticosteroid production, have also been proposed [22].

Type A behavior is also thought to stimulate the HPA axis and the sympathetic nervous system [23] and an association between type A behavior and hyperglycemia was reported in a few studies [24, 25]. Therefore it is possible that type A behavior is associated with the risk of diabetes; however, there are no papers that report this association as far as we know.

We found an inverse association between coffee consumption and the incidence of diabetes which was consistent with other studies. Similar inverse association between green tea consumption and the incidence of diabetes was also reported [26]. We also analyzed the effect of other beverages such as green tea, black tea or oolong tea; however, we found no clear association between the risk of diabetes and consumption of these beverages. These differences may come from the fact that these beverages contain less functional ingredients than coffee.

We also found a possible effect modification of perceived mental stress by coffee consumption, especially among men. Coffee consumption may have modified the effect of mental stress on diabetes. One possible explanation for the interaction between stress and coffee consumption is that coffee blocks cortisol formation by inhibiting $11 \beta$-hydroxysteroid dehydrogenase type 1 activity [27]. In addition, an effect modification between mental stress and coffee was reported in 
the case of blood pressure, that is, coffee blunts stressinduced blood pressure increase in habitual coffee drinkers [28]. However, further research on the mechanism of this effect modification, including whether it actually exists, is needed.

The strengths of our study were that (a) it was population-based; (b) it had a relatively large cohort size; (c) it had an adequate follow-up period; and (d) the study measured and included possible confounding factors. Nevertheless, our study also had several limitations. First, the assessment of diabetes mellitus was based on the results of a self-reported questionnaire. As mentioned in the methods section, however, 94\% of the diagnosed diabetes identified by this questionnaire were confirmed by medical records, and the sensitivity and specificity of the diagnosed diabetes were $82.6 \%$ and $99.7 \%$, respectively. Therefore we think that this assessment procedure was well validated. In previous studies $[17,29]$ we provided different numbers for these percentages based on the current (since 1999 in Japan) diagnostic criteria [30] to evaluate the usefulness of the self-reported method for detecting real diabetic conditions using the current diagnostic criteria. In this paper, diabetes was diagnosed according to the criteria at that time (year 1990) [18] and this method is appropriate for assessing the reporting validity of diabetes diagnosed in 1990 .

Second, perceived mental stress was assessed based on a single simple question, 'How much stress do you feel in your daily life?' The same question was used to investigate the association between mental stress and mortality from cardiovascular disease [31] and colorectal cancer [32]. Similar self-reported stress has been used to examine the association between stress and diseases such as breast cancer [33], stroke [34] and suicide [35]. However, more extensive stress evaluation may reveal the association between mental stress and the incidence of diabetes more precisely, which necessitates future studies.

Several sources of potential bias exist in our study. The follow-up bias does not seem to be important because the follow up percentage $(75 \%)$ was relatively high and no large differences in the major risk factors for diabetes were observed between the follow-up subjects and the lost-to-follow-up subjects at baseline (for stress, $16 \%$ and $64 \%$ of the follow-up subjects and $17 \%$ and $63 \%$ of the lost-to-follow-up subjects reported low and medium stress, respectively). Misclassification with regard to diabetes and exposure might also have caused biases. However, this type of bias in a cohort study generally distorts the results toward null.

Despite these limitations, as far as we know, our study is the first to demonstrate an association between psychological factors and the onset of diabetes in a large-scale cohort study.

\section{Acknowledgements}

We wish to thank Drs. Yoshihiko Takahashi and Tetsuya Mizoue for their critical reading of the manuscript and their helpful comments.

\section{Funding}

This study was supported by a grant-in-aid for Cancer Research H16-S2 and for the Third Term Comprehensive Ten-Year Strategy for Cancer Control H16010, and Health Sciences Research grants (Medical Frontier Strategy Research H13-008, Clinical Research for Evidence-based Medicine H14-008, H15-006, Comprehensive Research on Cardiovascular Diseases H16-019, H17-019, H18-028, H19-019) from the Ministry of Health, Labour and Welfare of Japan.

\section{Role of Funding Sources}

The sponsor of this study had no further role in study design; in the collection, analysis and interpretation of data; in the writing of the report; and in the decision to submit the paper for publication.

\section{Conflict of Interest}

All authors declare that they have no conflict of interest.

\section{Ethical Approval}

This study was approved by the institutional review board of the National Cancer Centre of Japan. 


\section{References}

1. Wild S, Roglic G, Green A, Sicree R, King H (2004) Global prevalence of diabetes: estimates for the year 2000 and projections for 2030. Diabetes Care 27: 1047-1053.

2. Zimmet P, Alberti KG, Shaw J (2001) Global and societal implications of the diabetes epidemic. Nature 414: 782-787.

3. Surwit RS, Schneider MS (1993) Role of stress in the etiology and treatment of diabetes mellitus. Psychosom Med 55: 380-393.

4. Surwit RS, Williams PG (1996) Animal models provide insight into psychosomatic factors in diabetes. Psychosom Med 58: 582-589.

5. Räikkönen K, Keltikangas-Järvinen L, Adlercreutz H, Hautanen A (1996) Psychosocial stress and the insulin resistance syndrome. Metabolism 45: 1533-1538.

6. Björntorp P, Holm G, Rosmond R (1999) Hypothalamic arousal, insulin resistance and Type 2 diabetes mellitus. Diabet Med 16: 373-383.

7. Rosmond R, Björntorp P (2000) The hypothalamic-pituitary-adrenal axis activity as a predictor of cardiovascular disease, type 2 diabetes and stroke. J Intern Med 247: 188-197.

8. VanItallie TB (2002) Stress: a risk factor for serious illness. Metabolism 51: 40-45.

9. Cobb S, Rose RM (1973) Hypertension, peptic ulcer, and diabetes in air traffic controllers. JAMA 224: 489492.

10. Kawakami N, Araki S, Takatsuka N, Shimizu H, Ishibashi H (1999) Overtime, psychosocial working conditions, and occurrence of non-insulin dependent diabetes mellitus in Japanese men. $J$ Epidemiol Community Health 53: 359-363.

11. Mooy JM, de Vries H, Grootenhuis PA, Bouter LM, Heine RJ (2000) Major stressful life events in relation to prevalence of undetected type 2 diabetes: the Hoorn Study. Diabetes Care 23: 197-201.

12. Agardh EE, Ahlbom A, Andersson T, Efendic S, Grill V, Hallqvist J, et al. (2003) Work stress and low sense of coherence is associated with type 2 diabetes in middleaged Swedish women. Diabetes Care 26: 719-724.

13. Goodwin RD, Davidson JR (2005) Self-reported diabetes and posttraumatic stress disorder among adults in the community. Prev Med 40: 570-574.

14. Tsugane S, Sobue T (2001) Baseline survey of JPHC study--design and participation rate. Japan Public Health Center-based Prospective Study on Cancer and Cardiovascular Diseases. J Epidemiol 11: S24-29.

15. Tsubono Y, Kobayashi M, Sasaki S, Tsugane S (2003) Validity and reproducibility of a self-administered food frequency questionnaire used in the baseline survey of the JPHC Study Cohort I. J Epidemiol 13: S125-133.

16. Ikeda A, Iso H, Kawachi I, Inoue M, Tsugane S (2008)
Type A behaviour and risk of coronary heart disease: The JPHC Study. Int J Epidemiol 37: 1395-1405.

17. Waki K, Noda M, Sasaki S, Matsumura Y, Takahashi Y, Isogawa A, et al. (2005) Alcohol consumption and other risk factors for self-reported diabetes among middleaged Japanese: a population-based prospective study in the JPHC study cohort I. Diabet Med 22: 323-331.

18. Kosaka K (1994) Diagnostic criteria for diabetes mellitus in Japan--from a report of the Japan Diabetes Society (JDS) Committee on the Diagnosis of Diabetes Mellitus, 1982. Diabetes Res Clin Pract 24 Suppl: S59-62.

19. Chrousos GP (2000) The role of stress and the hypothalamic-pituitary-adrenal axis in the pathogenesis of the metabolic syndrome: neuro-endocrine and target tissue-related causes. Int J Obes Relat Metab Disord 24 Suppl 2: S50-55.

20. Papanicolaou DA, Wilder RL, Manolagas SC, Chrousos GP (1998) The pathophysiologic roles of interleukin-6 in human disease. Ann Intern Med 128: 127-137.

21. Kiecolt-Glaser JK, Preacher KJ, MacCallum RC, Atkinson C, Malarkey WB, Glaser R (2003) Chronic stress and age-related increases in the proinflammatory cytokine IL-6. Proc Natl Acad Sci U S A 100: 90909095.

22. Pickup JC (2004) Inflammation and activated innate immunity in the pathogenesis of type 2 diabetes. Diabetes Care 27: 813-823.

23. Rozanski A, Blumenthal JA, Davidson KW, Saab PG, Kubzansky L (2005) The epidemiology, pathophysiology, and management of psychosocial risk factors in cardiac practice: the emerging field of behavioral cardiology. J Am Coll Cardiol 45: 637-651.

24. Räikkönen K, Keltikangas-Järvinen L, Hautanen A (1994) The role of psychological coronary risk factors in insulin and glucose metabolism. J Psychosom Res 38: 705-713.

25. Shen BJ, Countryman AJ, Spiro A, 3rd, Niaura R (2008) The prospective contribution of hostility characteristics to high fasting glucose levels: the moderating role of marital status. Diabetes Care 31: 1293-1298.

26. Iso H, Date C, Wakai K, Fukui M, Tamakoshi A (2006) The relationship between green tea and total caffeine intake and risk for self-reported type 2 diabetes among Japanese adults. Ann Intern Med 144: 554-562.

27. Atanasov AG, Dzyakanchuk AA, Schweizer RA, Nashev LG, Maurer EM, Odermatt A (2006) Coffee inhibits the reactivation of glucocorticoids by 11 betahydroxysteroid dehydrogenase type 1: a glucocorticoid connection in the anti-diabetic action of coffee? FEBS Lett 580: 4081-4085.

28. Sudano I, Spieker L, Binggeli C, Ruschitzka F, Luscher TF, Noll G, et al. (2005) Coffee blunts mental stress- 
induced blood pressure increase in habitual but not in nonhabitual coffee drinkers. Hypertension 46: 521-526.

29. Inoue M, Iwasaki M, Otani T, Sasazuki S, Noda M, Tsugane S (2006) Diabetes mellitus and the risk of cancer: results from a large-scale population-based cohort study in Japan. Arch Intern Med 166: 1871-1877.

30. Kuzuya T, Nakagawa S, Satoh J, Kanazawa Y, Iwamoto Y, Kobayashi M, et al. (2002) Report of the Committee on the classification and diagnostic criteria of diabetes mellitus. Diabetes Res Clin Pract 55: 65-85.

31. Iso H, Date C, Yamamoto A, Toyoshima H, Tanabe N, Kikuchi S, et al. (2002) Perceived mental stress and mortality from cardiovascular disease among Japanese men and women: the Japan Collaborative Cohort Study for Evaluation of Cancer Risk Sponsored by Monbusho (JACC Study). Circulation 106: 1229-1236.
32. Kojima M, Wakai K, Tokudome S, Tamakoshi K, Toyoshima H, Watanabe Y, et al. (2005) Perceived psychologic stress and colorectal cancer mortality: findings from the Japan Collaborative Cohort Study. Psychosom Med 67: 72-77.

33. Nielsen NR, Zhang ZF, Kristensen TS, Netterstrom B, Schnohr P, Gronbaek M (2005) Self reported stress and risk of breast cancer: prospective cohort study. $B M J$ 331: 548 .

34. Truelsen T, Nielsen N, Boysen G, Gronbaek M (2003) Self-reported stress and risk of stroke: the Copenhagen City Heart Study. Stroke 34: 856-862.

35. Feskanich D, Hastrup JL, Marshall JR, Colditz GA, Stampfer MJ, Willett WC, et al. (2002) Stress and suicide in the Nurses' Health Study. J Epidemiol Community Health 56: 95-98. 